\title{
KBNN Based on Coarse Mesh to Optimize the EBG Structures
}

\author{
Yi Chen, ${ }^{1}$ Yu-bo Tian, ${ }^{1}$ and Fei-yan Sun ${ }^{1,2}$ \\ ${ }^{1}$ School of Electronics and Information, Jiangsu University of Science and Technology, Zhenjiang, Jiangsu 212003, China \\ ${ }^{2}$ Nanjing Software Institute, Jinling Institute of Technology, Nanjing, Jiangsu 211169, China \\ Correspondence should be addressed to Yu-bo Tian; tianyubo@just.edu.cn
}

Received 11 July 2016; Revised 22 December 2016; Accepted 11 January 2017; Published 2 February 2017

Academic Editor: Shih Yuan Chen

Copyright (C) 2017 Yi Chen et al. This is an open access article distributed under the Creative Commons Attribution License, which permits unrestricted use, distribution, and reproduction in any medium, provided the original work is properly cited.

\begin{abstract}
The microwave devices are usually optimized by combining the precise model with global optimization algorithm. However, this method is time-consuming. In order to optimize the microwave devices rapidly, the knowledge-based neural network (KBNN) is used in this paper. Usually, the a priori knowledge of KBNN is obtained by the empirical formulas. Unfortunately, it is difficult to derive the corresponding formulas for the most electromagnetic problems, especially for complex electromagnetic problems; the formula derivation is almost impossible. We use precise mesh model of EM analysis as teaching signal and coarse mesh model as a priori knowledge to train the neural network (NN) by particle swarm optimization (PSO). The NN constructed by this method is simpler than traditional $\mathrm{NN}$ in structure which can replace precise model in optimization and reduce the computing time. The results of electromagnetic band-gap (EBG) structures optimally designed by this kind of KBNN achieve increase in the bandwidth and attenuation of the stopband and small passband ripple level which shows the advantages of the proposed KBNN method.
\end{abstract}

\section{Introduction}

The electromagnetic band-gap (EBG) $[1,2]$ is a kind of artificial periodic structure that prohibits the propagation of electromagnetic waves in certain frequency bands at microwave frequencies. Because of the unique feature of EBG structures, they have been applied to microwave circuits such as filters [3], power amplifiers [4], and antennas [5] to improve their performance.

Recently, neural network (NN) is widely used in microwave modeling and design [6-8] for its good learning ability and generalization. Usually, $\mathrm{NN}$ suffers a large number of samples to ensure the accuracy, which greatly increases the workload to establish NN. In existing papers, one of the effective ways to solve this problem is knowledge-based neural network (KBNN) [9]. The a priori knowledge of KBNN is always the empirical formula which contains the basic information about the microwave circuits but cannot achieve the required precision. There are different kinds of the knowledge-based structures, such as difference method [10], the a priori knowledge input (PKI) method [11], and KBNN [12]. In the existing methods, the a priori knowledge is obtained by empirical formula or NN $[13,14]$. If empirical formula is considered as a priori knowledge, the cost of calculation can be negligible, but not all microwave devices have equivalent circuit; if the a priori knowledge is obtained by $\mathrm{NN}$, the training of $\mathrm{NN}$ requires a large number of samples: both of them are flawed. In this paper, the a priori knowledge is obtained by coarse mesh model and is used as knowledge neurons in the hidden layers of NN. The advantage of this approach is that it can be widely applied to quick electromagnetic optimization even for the complex microwave devices. The method to build the KBNN model is given in the second section, including the neural network structure, acquisition of samples, and training method. In the third section, the KBNN is applied to tapered dual-plane EBG and papilionaceous dual-plane EBG which shows the feasibility of the method. Summary and discussion of future work are given in Section 4.

\section{The Proposed KBNN}

Assume that the structure of $\mathrm{KBNN}$ is $n \times m \times 1$, shown in Figure 1. Let $x$ represent input vector containing physical parameters of a microwave device, and let $y$ represent output vector of the KBNN. The number of knowledge-based 


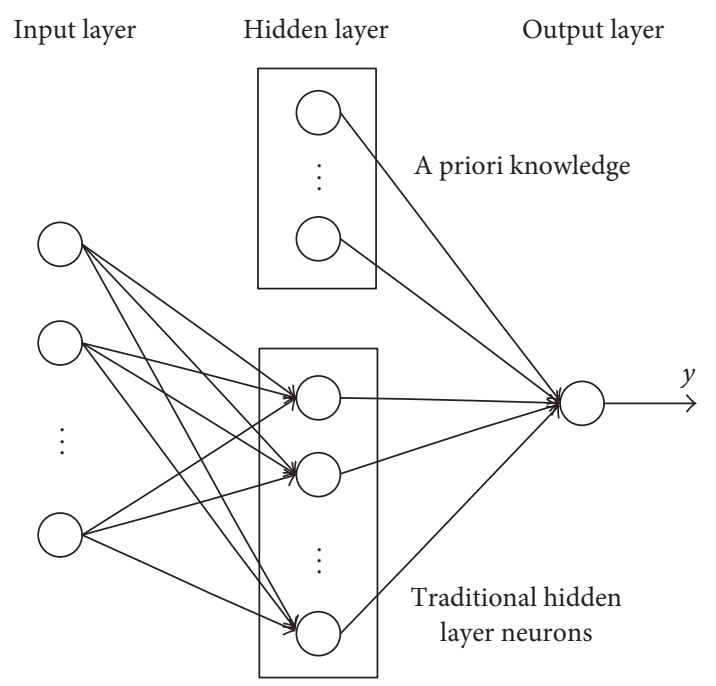

FIGURE 1: Structure of the proposed KBNN.

neurons in the hidden layer is $p$; $h k$ represent vector of the knowledge-based neurons values. The number of traditional hidden neurons is $q(p+q=m)$; $h$ represent output vector of traditional hidden neurons. Because no empirical formula is used here, there is no connection between input neurons and knowledge-based neurons. In short, the network combines the features of DC model and KBNN model; a part of the hidden neurons is used to generate the a priori knowledge and the other part is same as the traditional hidden neurons which are used to generate the difference between the coarse and fine models.

Given input $x$, the output can be computed by

$$
y=\sum_{j=1}^{p} \omega_{j} h k_{j}+\sum_{k=1}^{q} \omega_{k} h_{k}+\theta
$$

where $\omega_{j}$ is the weight between knowledge-based neurons and output layer, $\omega_{k}$ is the weight between traditional hidden neurons and output layer, and $\theta$ is threshold. $h_{k}$ can be computed by

$$
\begin{aligned}
& h_{k}=g\left(\gamma_{k}\right), \\
& \gamma_{k}=\sum_{i=1}^{n} \omega_{i k} x_{i}+\theta_{k},
\end{aligned}
$$

where $\omega_{i k}$ is the weight between input layer and traditional hidden neurons; $g(\cdot)$ is an activation function; we choose the sigmoid function (the function's gain $\lambda=1$ ) here.

$$
g(\gamma)=\frac{1}{1+e^{-\gamma}}
$$

In the simulation examples, both substrates have low loss. So we can approximatively consider the relationship between $\left|S_{21}\right|$ and $\left|S_{11}\right|$ as follows:

$$
\left|S_{11}\right|^{2}+\left|S_{21}\right|^{2}=1
$$

TABLE 1: Orthogonal table with 6 factors and 5 levels.

\begin{tabular}{lllllll}
\hline Number & A & B & C & D & E & F \\
\hline 1 & 1 & 1 & 1 & 4 & 1 & 1 \\
2 & 1 & 2 & 2 & 3 & 5 & 4 \\
3 & 1 & 3 & 3 & 2 & 4 & 2 \\
4 & 1 & 4 & 5 & 5 & 3 & 3 \\
5 & 1 & 5 & 4 & 1 & 2 & 5 \\
6 & 2 & 1 & 2 & 5 & 4 & 5 \\
7 & 2 & 2 & 3 & 1 & 3 & 1 \\
8 & 2 & 3 & 5 & 4 & 2 & 4 \\
9 & 2 & 4 & 4 & 3 & 1 & 2 \\
10 & 2 & 5 & 1 & 2 & 5 & 3 \\
11 & 3 & 1 & 3 & 3 & 2 & 3 \\
12 & 3 & 2 & 5 & 2 & 1 & 5 \\
13 & 3 & 3 & 4 & 5 & 5 & 1 \\
14 & 3 & 4 & 1 & 1 & 4 & 4 \\
15 & 3 & 5 & 2 & 4 & 3 & 2 \\
16 & 4 & 1 & 5 & 1 & 5 & 2 \\
17 & 4 & 2 & 4 & 4 & 4 & 3 \\
18 & 4 & 3 & 1 & 3 & 3 & 5 \\
19 & 4 & 4 & 2 & 2 & 2 & 1 \\
20 & 4 & 5 & 3 & 5 & 1 & 4 \\
21 & 5 & 1 & 4 & 2 & 3 & 4 \\
22 & 5 & 2 & 1 & 5 & 2 & 2 \\
23 & 5 & 3 & 2 & 1 & 1 & 3 \\
24 & 5 & 4 & 3 & 4 & 5 & 5 \\
25 & 5 & 5 & 5 & 3 & 4 & 1 \\
\hline & & & & & &
\end{tabular}

Because of this relationship, the number of knowledge-based neurons in hidden layer can be set from 0 to 2 . When the number is 0 , it means there is no knowledge-based neuron. $\left|S_{21}\right|$ is used as knowledge-based neuron when the number is 1 ; while it becomes two, both $\left|S_{11}\right|$ and $\left|S_{21}\right|$ are used.

The design parameters are generated by partial orthogonal experimental design to reduce the number of training samples. Orthogonal experiment design is a kind of design method to study multifactors and multilevels. It is based on orthogonality to select some representative points which is uniformly dispersed from the comprehensive experiment. In this paper, 6-factor and 5-level orthogonal table is used, shown in Table 1 . In the table, $A \sim F$ represent 6 design parameters and $1 \sim 5$ represent the levels of parameters. There are 25 groups of design parameters. If the training samples are too few, the KBNN cannot accurately map the relationship between input and output.

When the design parameters are confirmed, the output of KBNN is obtained by HFSS. Generally, the accuracy of HFSS simulation depends on adaptive analysis parameters, which are maximum number of passes and maximum delta $S$. The maximum number of passes value is the maximum number of mesh refinement cycles that you would like HFSS to perform. And delta $S$ is the magnitude of the change of $S$-parameters between two consecutive passes. They are stopping criterion for the adaptive solution. Usually, the maximum number of passes is 6 and maximum delta $S$ is 0.02 which can get 
accurate results. In the paper, delta $S$ is 0.3 in this case; that is, the maximum number of passes is 1 or 2 for coarse mesh model. When delta $S$ is about 0.02 , the maximum number of passes is 6 , which can be considered as fine mesh model. So we can find that if the mesh is not coarse enough, the simulation results will be closer to the accurate results. However, its analysis time will be much longer. So we just consider that the maximum number of passes of model is 1 or 2 which is suitable for a priori knowledge. VBScript is used here to provide an interface between HFSS and MATLAB. Thus, the data exchange can be realized which makes the acquisition of samples more automated and concise.

Particle swarm optimization (PSO), which is a kind of global optimization method [15], is chosen to train the network which can effectively avoid the local optimum of the NN. A flowchart of the proposed KBNN is shown in Figure 2. The number of particles is 80 with 1000 iterations for training the KBNN. We can judge the accuracy of the network by calculating the mean absolute error (MAE) of the sample and network correlation coefficient (NCC):

$$
\begin{aligned}
\text { MAE } & =\frac{\sum_{i=1}^{P}\left|y_{i}-s_{i}\right|}{P}, \\
\mathrm{NCC} & =\frac{\sum\left(s_{i}-\bar{s}\right)\left(y_{i}-\bar{y}\right)}{\sqrt{\sum\left(s_{i}-\bar{s}\right)^{2}\left(y_{i}-\bar{y}\right)^{2}}},
\end{aligned}
$$

where $s$ represents the corresponding result of HFSS simulation, $P$ is the total number of data samples, $\bar{s}$ is mean of the desired outputs, and $\bar{y}$ is mean of network outputs. NCC is an important standard to measure the rationality of the network. If it is closer to 1 , the network is more reasonable. Otherwise, the network needs to be trained again. According to Figure 2, we can establish the KBNN and use the KBNN to optimize microwave devices. A flowchart of the optimization by PSO is shown in Figure 3. The criterion of terminating the PSO for training KBNN and optimization is same. When the iteration reaches the maximum or the fitness meets the required error, the PSO can be terminated.

\section{Simulation Examples of EBG Structures}

3.1. Tapered Dual-Plane EBG Structure. The proposed KBNN is applied to a tapered dual-plane EBG [16], illustrated in Figure 4. As can be seen in Figure 4, the tapered dual-plane EBG consists of two single-plane EBG structures, one of which is a modulated microstrip line, while the other is a ground plane with etched circles. Between these two planes, there is a substrate with thickness $H=0.762 \mathrm{~mm}$, relative dielectric constant $\varepsilon_{r}=2.43$, and loss tangent $\tan D=0.0015$. The distance between the centers of two adjacent circles is $a_{1}=10.35 \mathrm{~mm}$; the radius of the circle from the middle to the side is $r_{1}, r_{2}$, and $r_{3}$. The distance between the centers of two adjacent squares is $a_{2}=10.35 \mathrm{~mm}$; the length of the square from the middle to the side is $l_{1}, l_{2}$, and $l_{3}$. Before optimization, $r_{1}=r_{2}=r_{3}=2 \mathrm{~mm}$ and $l_{1}=l_{2}=l_{3}=4 \mathrm{~mm}$. The frequency range is $1.7 \mathrm{GHz} \sim 4.3 \mathrm{GHz}$ with 56 sampled points.

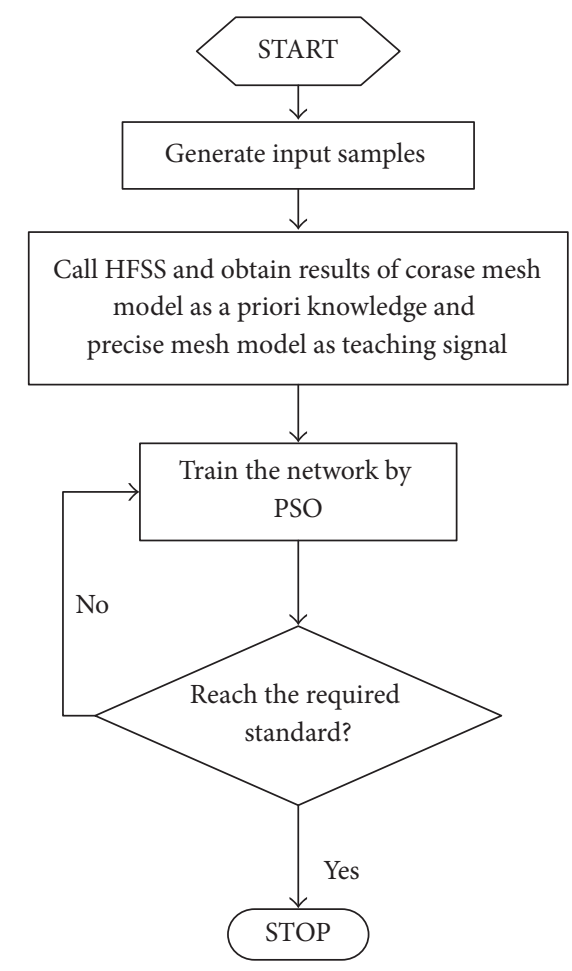

Figure 2: Flowchart of the proposed KBNN.

The simulated $S_{21}$ parameters of the tapered dual-plane EBG before optimization are shown in Figure 5. As can be seen in Figure 5, the tapered dual-plane EBG shows a $-10 \mathrm{~dB}$ bandwidth of $4.9029 \mathrm{GHz}$ with attenuation of $33.5691 \mathrm{~dB}$ at $9.6 \mathrm{GHz}$. The ripple level is $1.8247 \mathrm{~dB}$ in the lower passband and $6.3265 \mathrm{~dB}$ in the higher passband. To achieve maximum band-gap, the tapered dual-plane EBG is optimized by proposed KBNN.

First, we need to obtain the training samples to train the KBNN. The design parameter is $\mathbf{v}=\left[\begin{array}{llllll}l_{1} & l_{2} & l_{3} & r_{1} & r_{2} & r_{3}\end{array}\right]$. The ranges of them are as follows: $l_{1} \in[4,5], l_{2} \in[3.5,4.5]$, $l_{3} \in[3,4], \quad r_{1} \in[2,3], \quad r_{2}, r_{3} \in[1.5,2.5]$. The input of the proposed KBNN is $\mathbf{x}=\left[\begin{array}{ll}\mathbf{v} & f\end{array}\right]^{T}$. As it is said above, we sample 25 groups of $x_{m}$ by partial orthogonal experimental design. Hence, there are totally $25 \times 56=1400$ training samples.

To some degree, the reliability of proposed KBNN depends on the number of hidden neurons and knowledgebased neurons. So we train the same test sample in different number of hidden neurons and knowledge-based neurons; the results are listed in Table 2.

In Table 2, $2 \mathrm{~K} 4 \mathrm{H}$ represents the fact that the number of hidden neurons is 4 , in which the number of knowledgebased neurons is 2 , and $1 \mathrm{~K} 5 \mathrm{H}$ represents the fact that the number of hidden neurons is 6 , in which the number of knowledge-based neurons is 1 , and so on. It can be seen from Table 2 that when the number of knowledge-based neurons is 2 , the number of hidden neurons has little influence on the results. When the number of knowledge-based neurons is 1 , NCC decreases from 0.9944 to 0.9933 , and MAE increases 


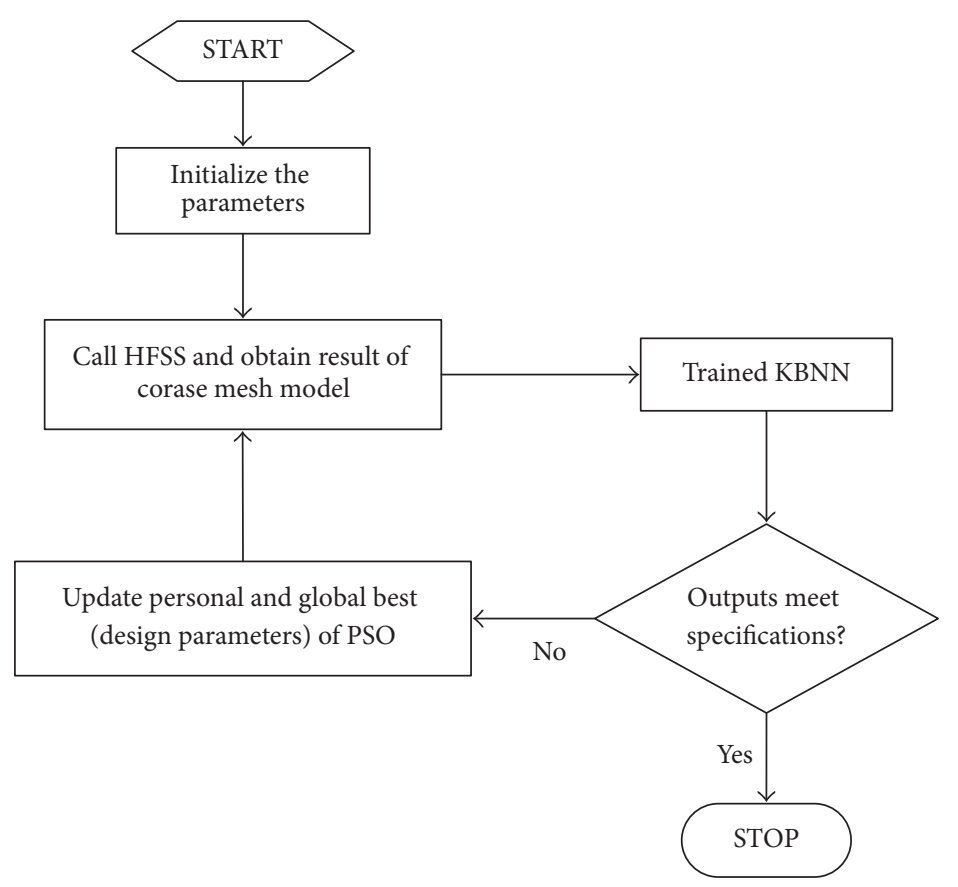

FIGURE 3: Flowchart of design optimization.

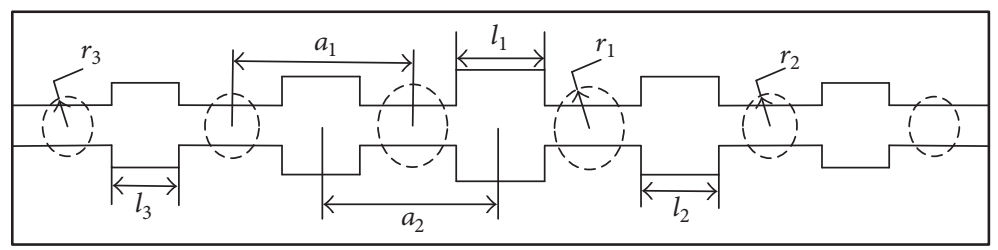

(a) Top view

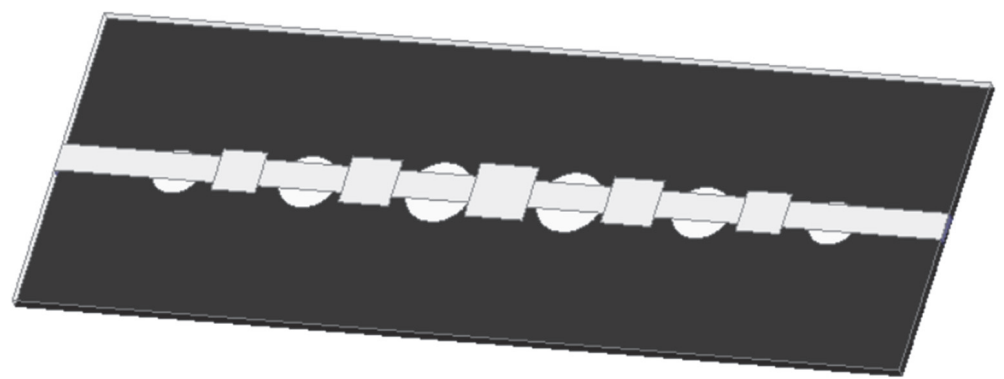

(b) HFSS model

FIGURE 4: Structure of tapered dual-plane EBG.

from 0.8040 to 1.1050 . When there is no knowledge-based neuron in hidden layer, the NN can be regarded as a PSONN. NCC drops to 0.9268 and MAE increases to 1.6115; the $\mathrm{NN}$ cannot map the relationship between input and output accurately. As a result, the existence of knowledgebased neurons can significantly improve the reliability of the network and make it easy to get the precise output.

We generate 5 different groups of test samples randomly and test on the $2 \mathrm{~K} 5 \mathrm{H} \mathrm{KBNN}$; the test results are shown in Table 3 and Figure 6. The KBNN is trained by PSO with 1000 iterations, while the 10-hidden-neuron multilayer perceptron (MLP) is trained with same training samples and 1500 iterations. As can be seen in Figure 6, the MAE of proposed KBNN is 0.8040 and the MAE of 10 -hidden-neuron MLP is 1.6733. Compared with MLP, the result of proposed KBNN is closer to the HFSS. In a short, in case of ensuring the accuracy, the proposed KBNN needs less number of training samples and has good generalization ability.

Use the trained $\mathrm{KBNN}$ with $2 \mathrm{~K} 5 \mathrm{H}$ structure to optimize the tapered dual-plane EBG. In the process of optimization, the precise model is replaced with KBNN which can reduce the time of optimization. The PSO algorithm to optimize has 


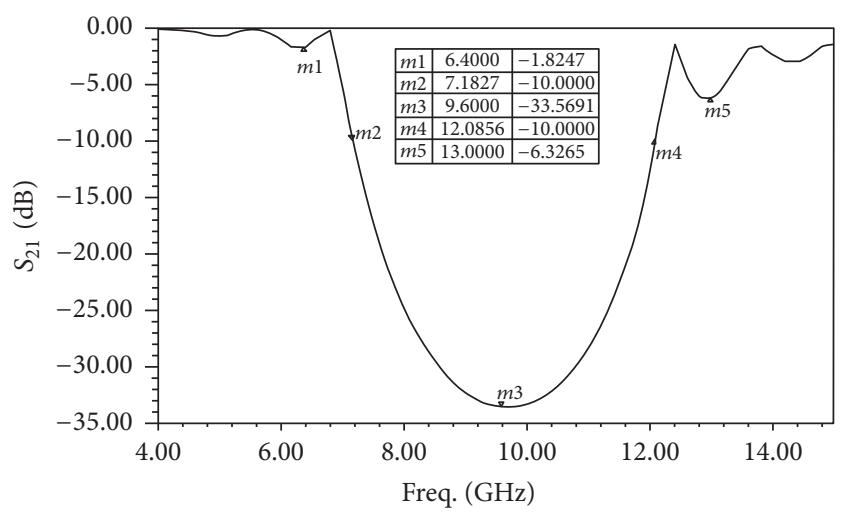

FIGURE 5: Simulated $S_{21}$ parameter of tapered dual-plane EBG.

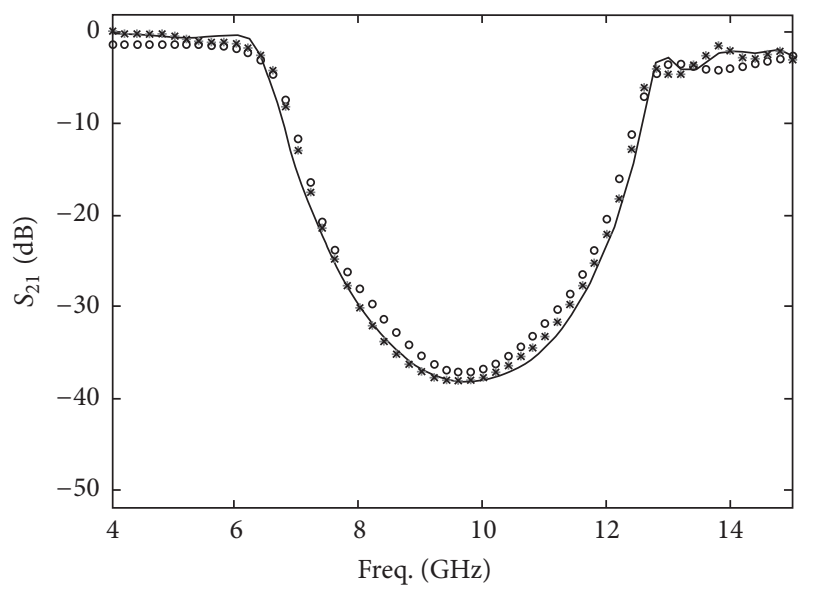

- Output of 10-hidden-layer MLP

* Output of proposed KBNN

— Result of HFSS simulation

FIgURE 6: Test sample result of tapered dual-plane EBG.

TABLE 2: Error in different hidden and knowledge neurons of tapered dual-plane EBG.

\begin{tabular}{lcc}
\hline Type of NN & MAE & NCC \\
\hline $2 \mathrm{~K} 4 \mathrm{H}$ & 1.0489 & 0.9936 \\
$2 \mathrm{~K} 5 \mathrm{H}$ & 0.8040 & 0.9944 \\
$2 \mathrm{~K} 6 \mathrm{H}$ & 0.9343 & 0.9943 \\
$2 \mathrm{~K} 7 \mathrm{H}$ & 0.7311 & 0.9938 \\
$2 \mathrm{~K} 8 \mathrm{H}$ & 0.8147 & 0.9944 \\
$2 \mathrm{~K} 9 \mathrm{H}$ & 0.7781 & 0.9941 \\
$2 \mathrm{~K} 10 \mathrm{H}$ & 0.9561 & 0.9942 \\
$1 \mathrm{~K} 5 \mathrm{H}$ & 1.1050 & 0.9933 \\
$0 \mathrm{~K} 5 \mathrm{H}$ & 1.6115 & 0.9268 \\
\hline
\end{tabular}

TABLE 3: Error of different test samples of tapered dual-plane EBG.

\begin{tabular}{lccccc}
\hline Number & 1 & 2 & 3 & 4 & 5 \\
\hline MAE & 0.8040 & 0.7810 & 0.8783 & 0.8193 & 0.6801 \\
\hline
\end{tabular}

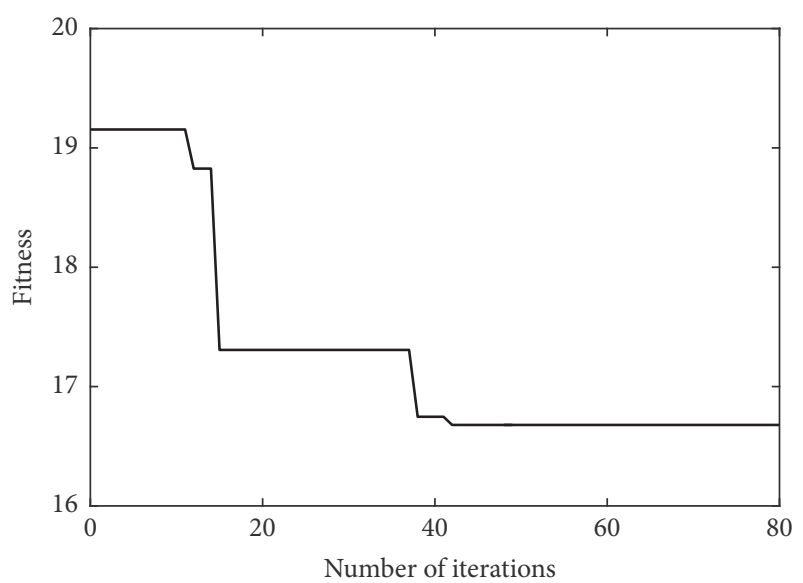

FIgURE 7: Fitness curve of optimization.

20 particles with 80 iterations. The output of KBNN is used to calculate the fitness. The fitness function is calculated by

$$
\begin{aligned}
\text { Fit } & =\min \left(5 \times y_{1}+y_{2}\right), \\
y_{1} & =\left|\min \left(y\left(f_{i}\right)\right)\right|, \quad i=1,2, \ldots, 10,50,51, \ldots, 56, \\
y_{2} & =|| \min \left(y\left(f_{i}\right)\right)|-45|, \quad i=20,21, \ldots, 38,
\end{aligned}
$$

where $f_{i}(i=1,2, \ldots, 56)$ is every point of frequency; $y\left(f_{i}\right)$ is the output of KBNN in corresponding frequency. Finally, we get the optimized sizes:

$$
=\left[\begin{array}{lllllll}
4.5929 & 3.9537 & 3.4882 & 2.6080 & 2.2631 & 1.8897
\end{array}\right] .
$$

The fitness curve is shown in Figure 7 and the results of optimization are shown in Table 4 and Figure 8.

It can be seen from optimization results that, after optimization, the maximum attenuation is from 33.5691 to $38.8096 \mathrm{~dB}$. The $10 \mathrm{~dB}$ bandwidth is 1.19 times wider than that before optimization. The ripple level is lowered from 1.8247 to $0.6878 \mathrm{~dB}$ in the lower passband and from 6.3265 to $4.0484 \mathrm{~dB}$ in the higher passband. This proves that it effective to use the proposed $\mathrm{KBNN}$ as precise model to optimize the tapered dual-plane EBG.

The average analysis time of coarse mesh model is about $35 \mathrm{~s}$, while the time of precise model is about $69 \mathrm{~s}$. When both coarse mesh model and precise model are analyzed, the time is about $86 \mathrm{~s}$. In the condition of 20 particles with 80 iterations, we can roughly calculate the total time of optimization by precise model in $69 \times 80 \times 20=110400 \mathrm{~s}$. The training process includes obtaining 25 sets training samples and training the KBNN. So the time of training process is calculated by $86 \times 25+696=2846 \mathrm{~s}$, and the total time of optimization by KBNN is $58846 \mathrm{~s}$. As can be seen from Table 5, although the proposed KBNN takes short time to obtain the training samples and train the $\mathrm{KBNN}$, the total time of optimization by KBNN is almost half of the time of optimization by precise model. As a result, using the KBNN based on coarse mesh to optimize the tapered dual-plane 
TABLE 4: Data of tapered dual-plane EBG before and after optimization.

\begin{tabular}{lcccc}
\hline & $\begin{array}{c}\text {-10 dB relative } \\
\text { bandwidth }\end{array}$ & $\begin{array}{c}\text { Maximum } \\
\text { attenuation/dB }\end{array}$ & $\begin{array}{c}\text { Ripple level in the } \\
\text { lower passband/dB }\end{array}$ & $\begin{array}{c}\text { Ripple level in the } \\
\text { higher passband/dB }\end{array}$ \\
\hline Before optimization & $51.07 \%$ & 33.5691 & 1.8247 & 6.3265 \\
After optimization & $60.88 \%$ & 38.8096 & 0.6878 & 4.0484 \\
\hline
\end{tabular}

TABLE 5: Time of optimization by precise model and proposed KBNN comparison.

\begin{tabular}{lcr}
\hline & Optimized by precise model & Optimized by proposed KBNN \\
\hline Training process & 0 & $86 \times 25+696=2846$ \\
Optimization & $69 \times 80 \times 20=110400$ & $35 \times 80 \times 20=56000$ \\
\hline Total & $110400=30.67 \mathrm{~h}$ & $58846=16.35 \mathrm{~h}$ \\
\hline
\end{tabular}

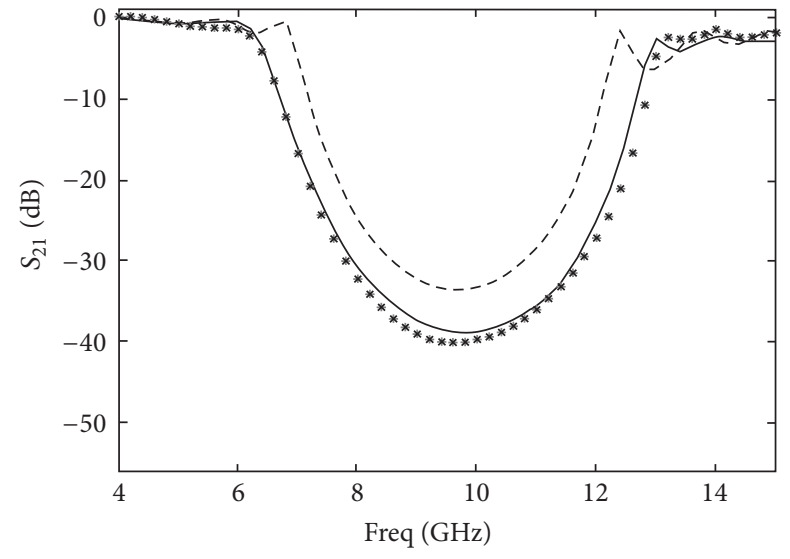

- - Result of HFSS simulation before optimization

* Output of proposed KBNN after optimization

— Result of HFSS simulation after optimization

FIGURE 8: Comparison of tapered dual-plane EBG before and after optimization.

EBG, to a great degree, shortens the time of optimization and makes it efficient.

3.2. Papilionaceous Dual-Plane EBG Structure. The proposed KBNN is applied to a papilionaceous dual-plane EBG [17], illustrated in Figure 9. As can be seen from Figure 9, the papilionaceous dual-plane EBG also consists of two singleplane EBG structures, one of which is six cells of papilionaceous patches, while the other is a ground plane with six etched rings. Between these two planes, there is a substrate with thickness $H=1.2 \mathrm{~mm}$, relative dielectric constant $\varepsilon_{r}=$ 3.38, and loss tangent $\tan D=0.0027$. The distance between the centers of two cells papilionaceous patches is $a_{1}=$ $17.6 \mathrm{~mm}$. The length of papilionaceous patch is $a_{2}=17.6 \mathrm{~mm}$. The widths of papilionaceous patch are, respectively, $w_{1}=$ $2.768 \mathrm{~mm}$ and $w_{2}=0.2 \mathrm{~mm}$. Before optimization, the inner and outer radii $r=R / 2=1.1 \mathrm{~mm}$. The frequency range is $3 \mathrm{GHz} 7.5 \mathrm{GHz}$ with 46 sampled points.

The simulated $S_{21}$ parameters of the papilionaceous dualplane EBG before optimization are shown in Figure 10. As can be seen from Figure 10, the papilionaceous dual-plane EBG shows a $-10 \mathrm{~dB}$ bandwidth of $2.4553 \mathrm{GHz}$ with attenuation of
TABLE 6: Error in different hidden and knowledge neurons of papilionaceous dual-plane EBG.

\begin{tabular}{lcc}
\hline Type of NN & MAE & NCC \\
\hline $2 \mathrm{~K} 4 \mathrm{H}$ & 1.1121 & 0.9867 \\
$2 \mathrm{~K} 5 \mathrm{H}$ & 0.9777 & 0.9879 \\
$2 \mathrm{~K} 6 \mathrm{H}$ & 0.9549 & 0.9864 \\
$2 \mathrm{~K} 7 \mathrm{H}$ & 0.9398 & 0.9880 \\
$2 \mathrm{~K} 8 \mathrm{H}$ & 0.9195 & 0.9869 \\
$2 \mathrm{~K} 9 \mathrm{H}$ & 0.8991 & 0.9881 \\
$2 \mathrm{~K} 10 \mathrm{H}$ & 0.9857 & 0.9882 \\
$1 \mathrm{~K} 7 \mathrm{H}$ & 1.0683 & 0.9870 \\
$0 \mathrm{~K} 7 \mathrm{H}$ & 9.2719 & 0.7471 \\
\hline
\end{tabular}

$32.5114 \mathrm{~dB}$ at $5.2 \mathrm{GHz}$. The ripple level is $3.648 \mathrm{~dB}$ in the lower passband and $5.3396 \mathrm{~dB}$ in the higher passband.

The design parameter of papilionaceous dual-plane EBG is $\mathbf{v}=\left[\begin{array}{llllll}R_{1} & R_{2} & R_{3} & r_{1} & r_{2} & r_{3}\end{array}\right]$. The subscript numbers are set from center to side. The relationship of them is $R_{1}=$ $2 r_{1}, R_{2}=2 r_{2}$, and $R_{3}=2 r_{3}$, The ranges of them are as follows: $R_{1}, R_{2}, R_{3} \in[1.5,4]$ The input of the proposed KBNN is $\mathbf{x}=\left[\begin{array}{ll}\mathbf{v} & f\end{array}\right]^{T} . \mathbf{v}$ has 25 groups. Hence, there are totally $25 \times 46=1150$ training samples.

The results on same test sample of different number of hidden neurons and knowledge-based neurons are listed in Table 6.

From the table, we know that when the number of knowledge-based neurons is 2 , the number of hidden neurons has little influence on the results. When the number of knowledge-based neurons is 1, NCC decreases from 0.9880 to 0.9870 , and MAE increases from 0.9398 to 1.0683 . When there is no knowledge-based neuron in hidden layer, the NCC drops to 0.7471 and MAE increases to 9.2719. We can draw same conclusions that the proposed KBNN makes the NN structure simpler and has easy access to accuracy value.

The test results on 5 different test samples of $2 \mathrm{~K} 7 \mathrm{H} \mathrm{KBNN}$ are shown in Table 7 and Figure 10. As can be seen from Figure 11, the MAE of the proposed KBNN is 0.9398 and the MAE of 10-hidden-neuron MLP is 1.2488. Similarly, the result of the proposed KBNN is closer to the HFSS compared with the MLP NN. The modeling of the papilionaceous dual-plane 


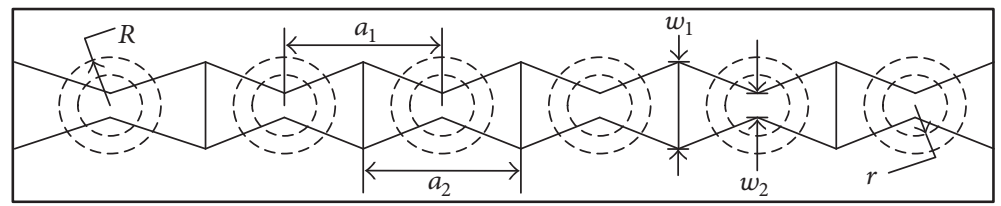

(a) Top view

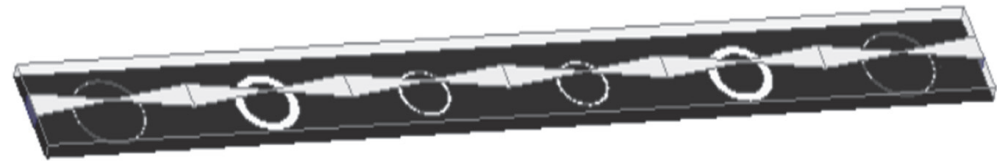

(b) HFSS model

FIGURE 9: Structure of papilionaceous dual-plane EBG.

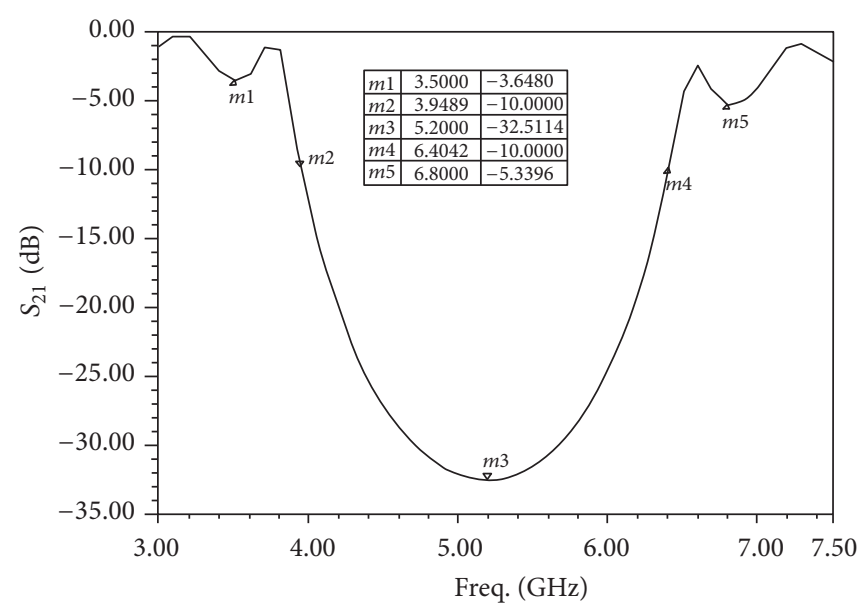

FIGURE 10: Simulated $S_{21}$ parameter of papilionaceous dual-plane EBG.

TABLE 7: Error of different test samples of papilionaceous dual-plane EBG.

\begin{tabular}{lccccc}
\hline Number & 1 & 2 & 3 & 4 & 5 \\
\hline MAE & 0.9398 & 0.8664 & 0.8757 & 1.0314 & 0.9369 \\
\hline
\end{tabular}

EBG shows same conclusion with tapered dual-plane EBG which verifies superiority of the proposed KBNN.

Same with the optimization of tapered dual-plane EBG, the papilionaceous dual-plane EBG is optimized by exploiting the trained $\mathrm{KBNN}$ with $2 \mathrm{~K} 7 \mathrm{H}$ structure. The fitness function is calculated by

$$
\begin{aligned}
\text { Fit } & =\min \left(10 \times y_{1}+y_{2}\right), \\
y_{1} & =\left|\min \left(y\left(f_{i}\right)\right)\right|, \quad i=1,2, \ldots, 6,41,42, \ldots, 46, \\
y_{2} & =|| \min \left(y\left(f_{i}\right)\right)|-40|, \quad i=7,8, \ldots, 40 .
\end{aligned}
$$

The optimized size is $\mathbf{v}$ $\left[\begin{array}{llllll}3.6590 & 3.0423 & 2.0134 & 1.8295 & 1.5211 & 1.0067\end{array}\right]$. The fitness curve is shown in Figure 12 and the results of optimization are shown in Figure 13 and Table 8.

It can be seen from the optimization results that, after optimization, the maximum attenuation is from 32.5144 to
$39.7025 \mathrm{~dB}$. The $10 \mathrm{~dB}$ bandwidth is 1.268 times wider than that before optimization. The ripple level is significantly lowered from 3.6480 to $1.6889 \mathrm{~dB}$ in the lower passband and from 5.3396 to $2.0679 \mathrm{~dB}$ in the higher passband. In this example, the proposed $\mathrm{KBNN}$ is also proven to be a good way to optimize the papilionaceous dual-plane EBG.

The average analysis time of coarse mesh model is about $23.8 \mathrm{~s}$, while the time of precise model is about $38.5 \mathrm{~s}$. When both coarse mesh model and precise model are analyzed, the time is about $58 \mathrm{~s}$. Similarly, we can calculate the total time of optimization by two ways, shown in Table 9. As can be seen from Table 9, using the KBNN based on coarse mesh to optimize the papilionaceous dual-plane EBG also shortens the time of optimization and makes it easy to optimize.

\section{Conclusion}

In this paper, we propose a new way to obtain the a priori knowledge by coarse mesh model which avoids complicated derivation of the formulas or the cost of obtaining a large number of samples. This method is also applied to those microwave devices which do not have empirical formulas. The modeling results of tapered dual-plane EBG and papilionaceous dual-plane EBG show that the knowledge-based 
TABLE 8: Data of papilionaceous dual-plane EBG before and after optimization.

\begin{tabular}{lcccc}
\hline & $\begin{array}{c}-10 \mathrm{~dB} \text { relative } \\
\text { bandwidth }\end{array}$ & $\begin{array}{c}\text { Maximum } \\
\text { attenuation/dB }\end{array}$ & $\begin{array}{c}\text { Ripple level in the } \\
\text { lower passband/dB }\end{array}$ & $\begin{array}{c}\text { Ripple level in the } \\
\text { higher passband/dB }\end{array}$ \\
\hline Before optimization & $47.22 \%$ & 32.5144 & 3.6480 & 5.3396 \\
After optimization & $59.87 \%$ & 39.7025 & 1.6889 & 2.0679 \\
\hline
\end{tabular}

TABLE 9: Time of optimization by precise model and proposed KBNN comparison.

\begin{tabular}{lcc}
\hline & Optimized by precise model & Optimized by proposed KBNN \\
\hline Training process & 0 & $58 \times 25+556=2006$ \\
Optimization & $38.5 \times 80 \times 20=61600$ & $23.8 \times 80 \times 20=38080$ \\
\hline Total & $61600=17.11 \mathrm{~h}$ & $40086=11.135 \mathrm{~h}$ \\
\hline
\end{tabular}

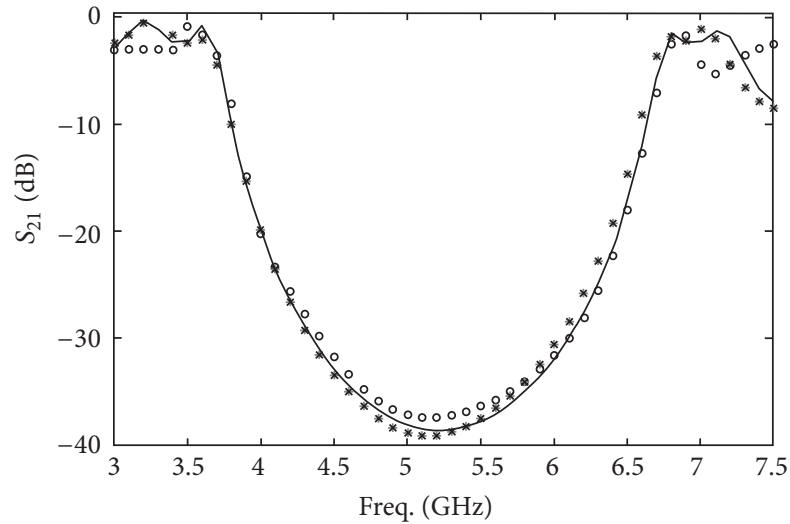

* Output of proposed KBNN

- Output of 10-hidden-layer MLP

— Result of HFSS simulation

FIGURE 11: Test sample result of papilionaceous dual-plane EBG.

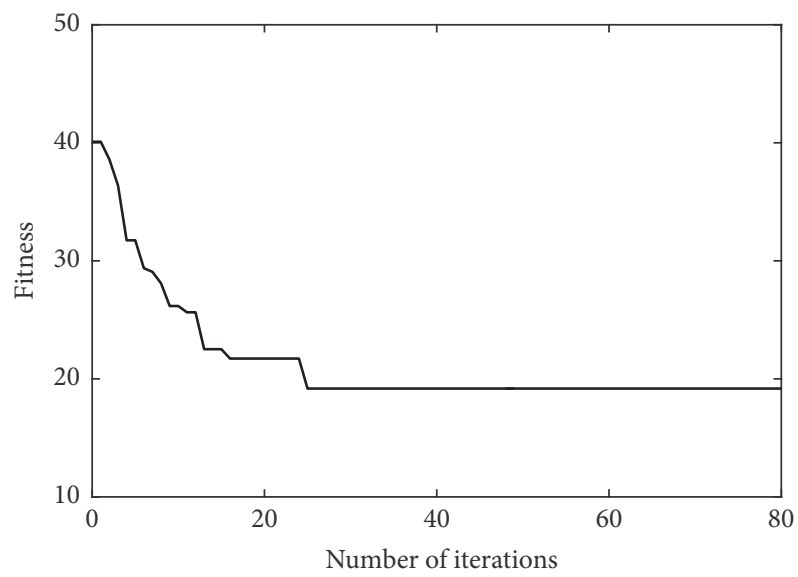

FIGURE 12: Fitness curve of optimization.

neurons in the hidden layer can significantly reduce the number of hidden neurons which makes the structure simpler and the test results are in good accordance with the results of HFSS simulation which shows the strong generalization ability of the proposed KBNN. The optimizations of above examples indicate that using the proposed KBNN to optimize the microwave devices is feasible which can obviously reduce

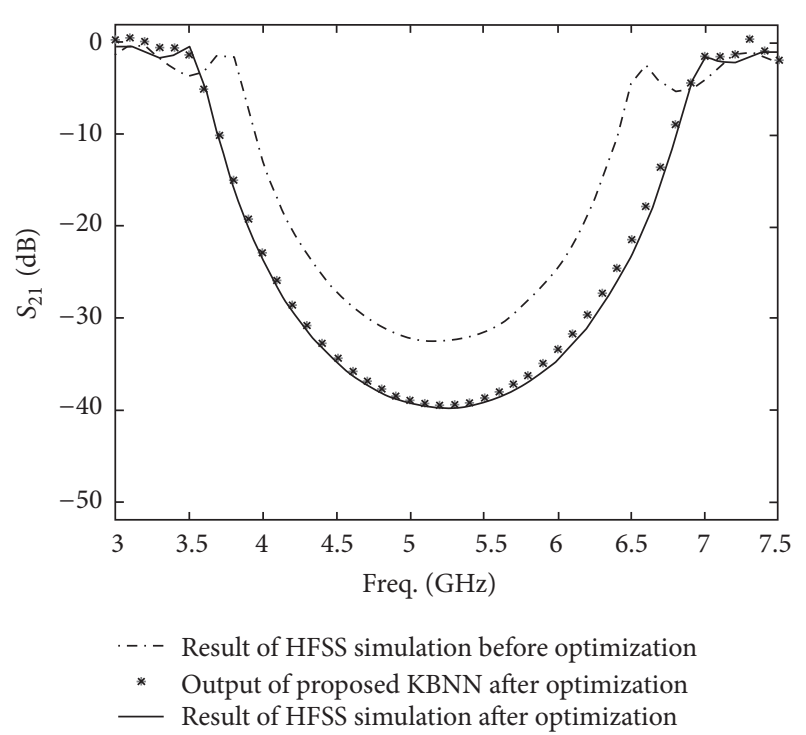

FIGURE 13: Comparison of papilionaceous dual-plane EBG before and after optimization.

the optimization time. In other words, the proposed KBNN has good value in the optimization of microwave devices. The other way to obtain the a priori knowledge will be investigated in the future work and the new structure of $\mathrm{KBNN}$ will also be considered.

\section{Competing Interests}

The authors declare that they have no competing interests.

\section{Acknowledgments}

This work is supported by Key Research and Development Program Project of Social Development in Jiangsu Province, China (no. BE2016723).

\section{References}

[1] E. Yablonovitch, "Inhibited spontaneous emission in solid-state physics and electronics," Physical Review Letters, vol. 58, no. 20, pp. 2059-2062, 1987. 
[2] S. John, "Strong localization of photons in certain disordered dielectric super lattice," Physical Review Letters, vol. 58, no. 20, pp. 2486-2488, 1987.

[3] D. A. Nesic and B. M. Kolundzija, "EBG band-stop filter with suppression of 3 spurious stop-bands," in Proceedings of the International Workshop on Antenna Technology: Small Antennas, Innovative Structures and Materials (iWAT '10), pp. 1-4, Lisbon, Portugal, March 2010.

[4] V. Radisic, Y. Qian, and T. Itoh, "Broad-band power amplifier using dielectric photonic bandgap structure," IEEE Microwave \& Guided Wave Letters, vol. 8, no. 1, pp. 13-14, 1998.

[5] S. Ghosh, T.-N. Tran, and T. Le-Ngoc, "Dual-layer EBG-based miniaturized multi-element antenna for MIMO systems," IEEE Transactions on Antennas \& Propagation, vol. 62, no. 8, pp. 3985-3997, 2014.

[6] H. Kabir, L. Zhang, M. Yu, P. H. Aaen, J. Wood, and Q.-J. Zhang, "Smart modeling of microwave devices," IEEE Microwave Magazine, vol. 11, no. 3, pp. 105-118, 2010.

[7] F. Mkadem and S. Boumaiza, "Extended hammerstein behavioral model using artificial neural networks," IEEE Transactions on Microwave Theory \& Techniques, vol. 57, no. 4, pp. 745-751, 2009.

[8] Z. Marinkovic, G. Crupi, G. Avolio, V. Markovic, A. Caddemi, and D. M. M.-P. Schreurs, "Neural network modelling of GaAs pHEMTs suitable for millimeter-wave mixer design," in Proceedings of the International Workshop on Integrated Nonlinear Microwave and Millimetre-wave Circuits (INMMiC '15), Taormina, Italy, October 2015.

[9] G. G. Towell and J. W. Shavlik, "Knowledge-based artificial neural networks," Artificial Intelligence, vol. 70, no. 1-2, pp. 119165, 1994.

[10] T. Khan and A. De, "Prediction of slot shape and slot size for improving the performance of microstrip antennas using knowledge-based neural networks," International Scholarly Research Notices, vol. 2014, Article ID 957469, 9 pages, 2014.

[11] C. Li, L. J. Xue, and J. Xu, "Knowledge-based artificial neural network models for microstrip radialstub," Chinese Journal of Electronics, vol. 29, no. 12, pp. 1696-1698, 2001 (Chinese).

[12] B.-Z. Wang, D. Zhao, and J. Hong, "Modeling stripline discontinuities by neural network with knowledge-based neurons," IEEE Transactions on Advanced Packaging, vol. 23, no. 4, pp. 692-698, 2000.

[13] W. C. Na and Q. J. Zhang, "Automated knowledge-based neural network modeling for microwave applications," IEEE Microwave \& Wireless Components Letters, vol. 24, no. 7, pp. 499-501, 2014.

[14] Z. Marinković, O. Pronić-Rančić, and V. Marković, "Smallsignal and noise modeling of class of HEMTs using knowledgebased artificial neural networks," International Journal of RF and Microwave Computer-Aided Engineering, vol. 23, no. 1, pp. 3439, 2013.

[15] T. Yubo, Hybrid Neural Network Techniques, Science Press, Beijing, China, 2009 (Chinese).

[16] S. Y. Huang and Y. H. Lee, "Tapered dual-plane compact electromagnetic bandgap microstrip filter structures," IEEE Transactions on Microwave Theory \& Techniques, vol. 53, no. 9, pp. 2656-2664, 2005.

[17] S. Sha, T. Peng, and Y. Tian, "Optimum design of double-layer EBG structure," Piezoelectrics \& Acoustooptics, vol. 33, no. 5, pp. 846-848, 2011. 


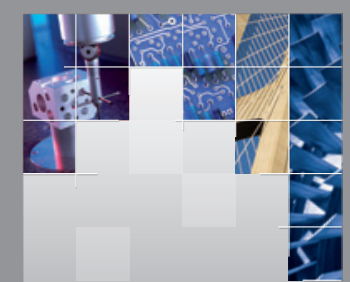

\section{Enfincering}
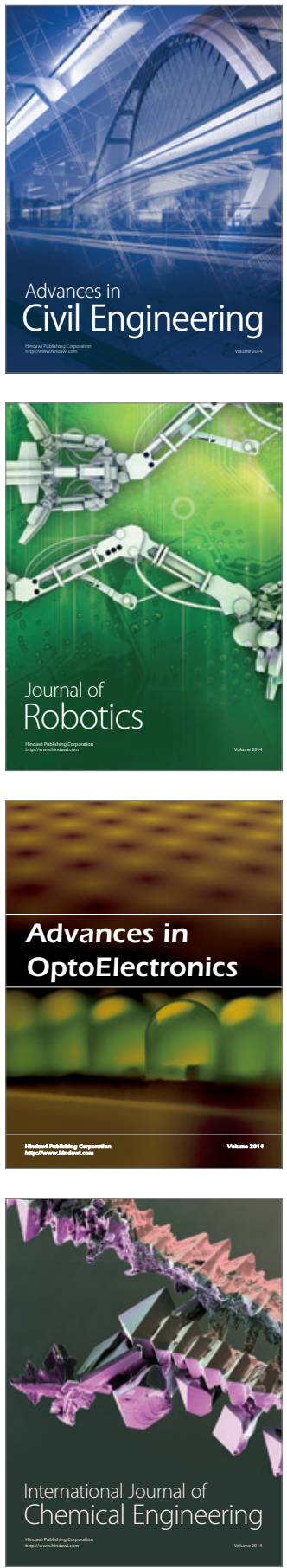

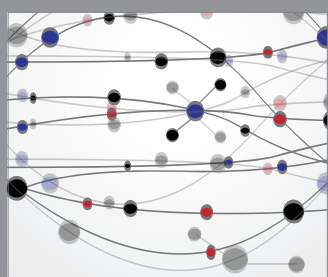

The Scientific World Journal

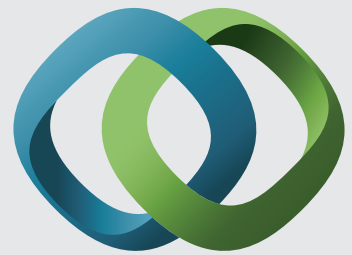

\section{Hindawi}

Submit your manuscripts at

https://www.hindawi.com
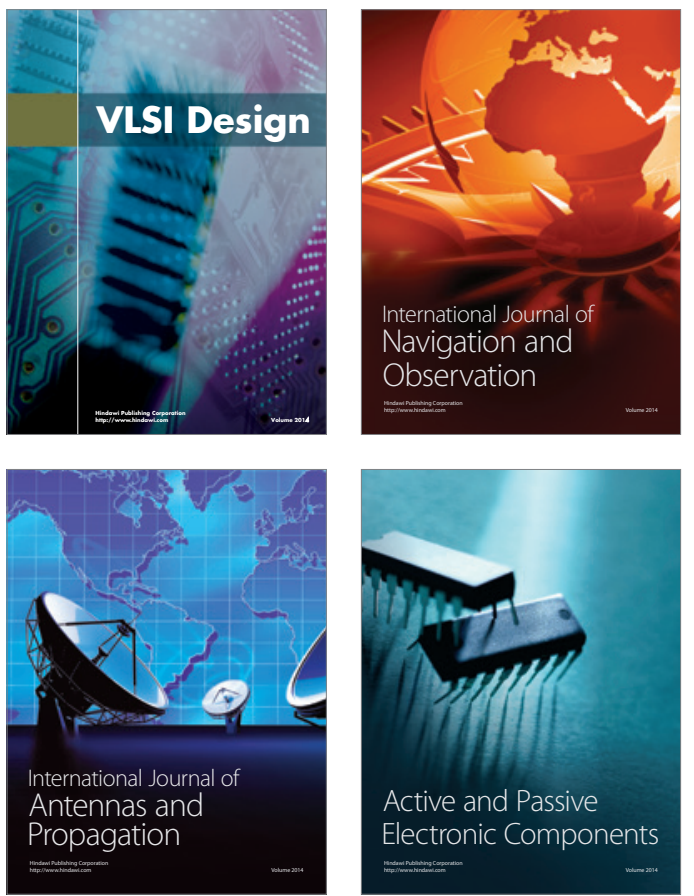
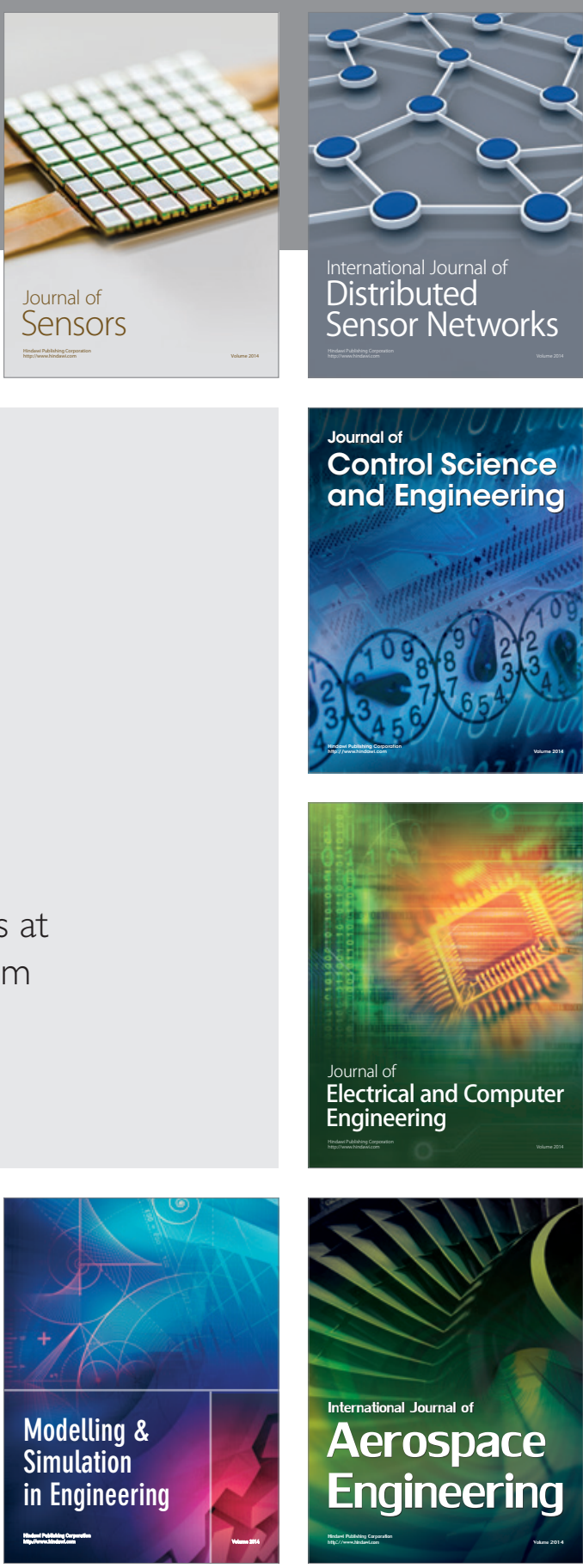

International Journal of

Distributed

Sensor Networks

$-$

Joumal of

Control Science

and Engineering
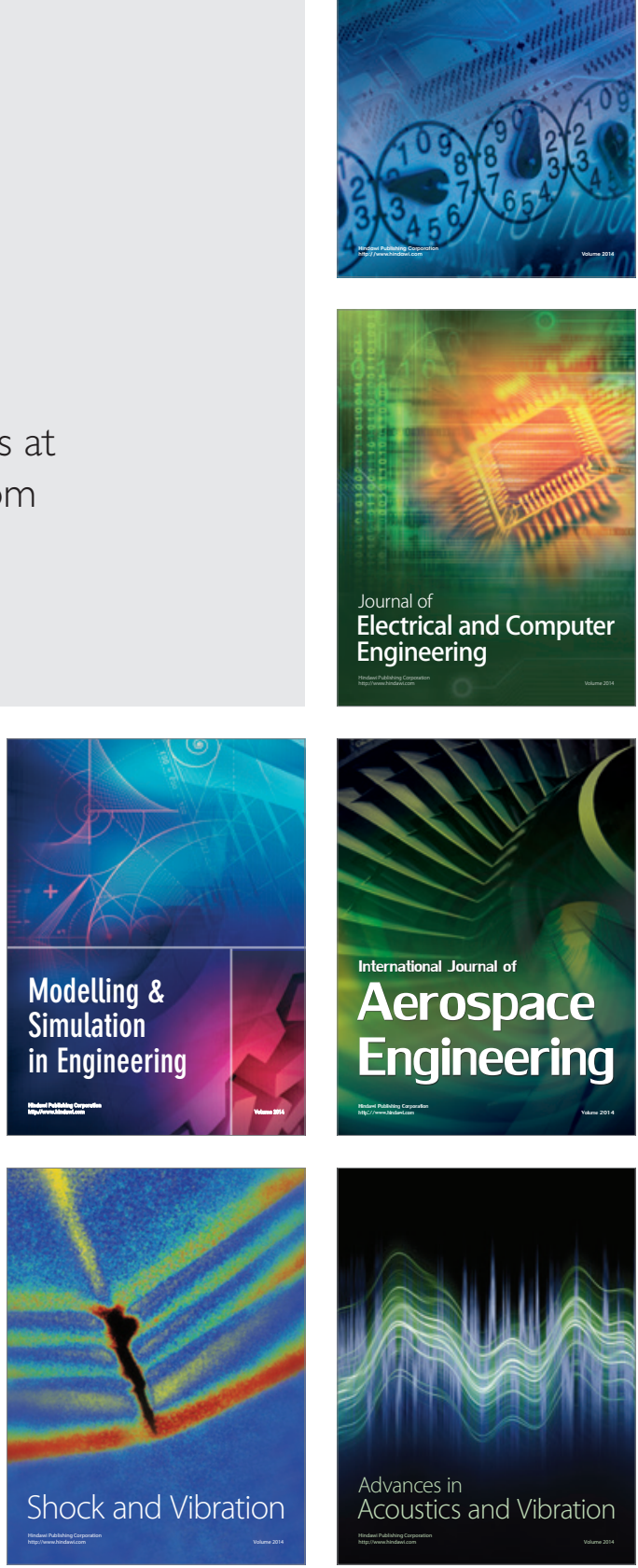